\section{Atypical hemolytic uremic syndrome secondary to lupus nephritis, responsive to eculizumab}

\author{
Alexander G. Raufi, ${ }^{1}$ Shruti Scott, ${ }^{1}$ \\ Omar Darwish, ${ }^{1}$ Kevin Harley, ${ }^{1}$ \\ Kanwarpal Kahlon, ${ }^{1}$ Sheetal Desai, ${ }^{1}$ \\ Yuxin Lu, ${ }^{2}$ Minh-Ha Tran² \\ 1Department of Internal Medicine, \\ 2Department of Pathology and \\ Laboratory Medicine, University of \\ California, Irvine, CA, USA
}

\begin{abstract}
Among the spectrum of disease manifestations associated with systemic lupus erythematosus, lupus nephritis is particularly concerning due to the potential for renal failure. This autoimmune attack may not, however, be limited to the kidney and is increasingly being recognized as a trigger for atypical Hemolytic Uremic Syndrome (aHUS). Atypical HUS falls under the spectrum of the thrombotic microangiopathies (TMAs) - a group of disorders characterized by microangiopathic hemolytic anemia, thrombocytopenia, and end organ damage. Although plasma exchange is considered first-line therapy for thrombotic thrombocytopenic purpura - a TMA classically associated with autoimmune depletion of ADAMTS-13 aHUS demonstrates less reliable responsiveness to this modality. Instead, use of the late complement inhibitor Eculizumab has emerged as an effective modality for the management of such patients. Diagnosis of aHUS, however, is largely clinically based, relying heavily upon a multidisciplinary approach. Herein we present the case of a patient with atypical HUS successfully treated with Eculizumab in the setting of Class IV-G (A) lupus nephritis and hypocomplementemia.
\end{abstract}

\section{Introduction}

Complement mediated TMA (also referred to as atypical Hemolytic Uremic Syndrome) is a rare (annual incidence 1:500,000) ) $^{1,2}$ entity belonging to the family of disorders collectively called thrombotic microangiopathies (TMAs). ${ }^{3}$ The pathophysiology of complement-mediated TMA involves unchecked activation of the alternative pathway leading to excessive deposition and deleterious action of complement on the endothelium, often with a predilection for the renal vasculature. ${ }^{4}$ Failure to control com- plement activation is due predominantly to inherited or acquired deficiencies of complement regulatory proteins - described in over $50 \%$ of individuals with complement mediated TMA. ${ }^{5,6}$ Known mutations leading to sporadic and recurrent complement mediated TMA include those that affect regulatory proteins that are both soluble (Factor I, which, in cooperation with Factor H, MCP and other proteins cleaves C4b and C3b; Factor $\mathrm{H}$, which binds C3b and disrupts the alternative pathway C3 convertase) and membrane bound (Membrane Cofactor Protein, CD46); a number of other defects have also been described. ${ }^{7}$ Patients with complement mediated TMA, even those in whom a clear genetic or antibody-mediated predilection is absent, may experience recurrences that may also damage renal allografts following kidney transplantation. ${ }^{5}$

Typically, complement mediated TMA will present as a microangiopathic hemolytic anemia, with thrombocytopenia, evidence of mechanical hemolysis (presence of schistocytosis on peripheral blood smear, increased $\mathrm{LDH}$, decreased haptoglobin), and varying degrees of end-organ dysfunction. ${ }^{2}$ Other manifestations include severe hypertension, central nervous system features (such as altered mental status, diplopia, or motor deficits), acute coronary syndrome due to cardiac microangiopathy, distal ischemic gangrene, and acute multi-organ failure. ${ }^{2}$

Responses to plasma-based therapeutics (either plasma infusion or plasma exchange) in complement-mediated TMA appears highest among those with MCP mutations (97\%) and lowest among those with CFI mutations (25\%) with 3 year outcomes of ESRD or death as high as 77\% among those with $\mathrm{CFH}$ and lowest among those with MCP mutations (6\%). ${ }^{5}$ Despite treatment responses to individual bouts of TMA, therefore, plasma based therapeutics are not uniformly effective.

Eculizumab, an inhibitor of human C5 complement protein, has emerged as an important therapeutic agent in the treatment of complement mediated TMA. This agent was studied by Legendre and colleagues in 37 patients with complement mediated TMA with or without thrombocytopenia. ${ }^{8}$ Eculizumab was dosed intravenously within 1-6 hours of most recent plasma therapy at a dose of $900 \mathrm{mg}$ per week for 4 weeks, then $1200 \mathrm{mg}$ on week 5 , then $1200 \mathrm{mg}$ every 2 weeks beginning week 6 . Patients continuing plasma based therapeutics during eculizumab treatment received $600 \mathrm{mg}$ eculizumab booster before plasma infusion or within 1 hour following completion of each plasma exchange. In the group with thrombocytopenia, median platelet-count improvements of $73 \times 10^{9} / \mathrm{L}$ from baseline occurred by week 26 of therapy; 4/5 patients discontinued dialysis. In the group without thrombocytopenia, $80 \%$ achieved TMA-free status.
Correspondence: Minh-Ha Tran, University of California, Irvine, UCIMC, Bldg. 1, Rm. 3003, Mail Code 4805, Irvine, CA 92697, USA.

Tel.: +1.714.456.8925.

E-mail:minhhat1@uci.edu

Key words: Atypical hemolytic uremic syndrome; Microangiopathic hemolytic anemia; Thrombotic microangiopathy; Lupus nephritis.

Contributions: the authors contributed equally.

Conflict of interest: the authors declare no potential conflict of interest.

Received for publication: 27 May 2016. Revision received: 11 September 2016. Accepted for publication: 23 September 2016.

This work is licensed under a Creative Commons Attribution-NonCommercial 4.0 International License (CC BY-NC 4.0).

(C) Copyright A.G. Raufi et al., 2016

Licensee PAGEPress, Italy

Hematology Reports 2016; 8:6625

doi:10.4081/hr.2016.6625

Here we present a unique case of aHUS which developed in a patient with newly diagnosed systemic lupus erythematosus (SLE), failed a course of therapeutic plasma exchange, but that subsequently responded to eculizumab.

\section{Case Report}

A 25 year-old Vietnamese female presented with a one month history of arthralgias, daily symmetric arthritis of the metacarpophalangeal (MCP), proximal interphalangeal (PIP), and metatarsophalangeal (MTP) joints accompanied by two hours of morning stiffness. In addition, she reported myalgias, facial swelling, intermittent nausea, vomiting, diarrhea, and generalized headaches associated with photo/phonosensitivity but no visual or focal neurologic deficits. Ten days prior to admission, she developed a mild, productive cough but no fever or chills. She also had dyspnea on exertion, one-pillow orthopnea, and intermittent pleuritic chest discomfort - most pronounced on deep inspiration. On exam she was noted to have a subtle malar rash and diffuse tenderness on abdominal exam.

Pertinent laboratory results include a positive antinuclear antibody (ANA; in an atypical speckled pattern suggestive of SSA/Ro) with a titer to >320; anti-double stranded DNA with a titer to $>5120$; positive ANCA but negative MPO and PR3 antibodies. Anti-Smith IgG was elevated at $31 \mathrm{U}(0-19)$, SSA (R0) was elevated 
at $120 \mathrm{U}(0-19)$ but SSB (LA) was normal range at $15 \mathrm{U}(0-19)$. Hypocomplementemia was noted with reduced levels of $\mathrm{C} 3$ and $\mathrm{C} 4-3$ $\mathrm{mg} / \mathrm{dL}$ (16-47) and $13.9 \mathrm{mg} / \mathrm{dL}$ (88-201)respectively.

Admission blood urea nitrogen (BUN) and creatinine $(\mathrm{Cr})$ were both elevated at $20 \mathrm{mg} / \mathrm{dL}$ (7-25) and $1.3 \mathrm{mg} / \mathrm{dL}$ (0.6-1.2). Urinalysis was positive for protein at $>500 \mathrm{mg} / \mathrm{dL}$, hemoglobinuria (LARGE), red blood cells 58/hpf, white blood cells $1 / \mathrm{hpf}$. Renal ultrasound demonstrated normal sized kidneys with increased echogenicity. A renal biopsy (Figure 1) performed on hospital day \#4 showed diffuse proliferative lupus nephritis, Class IV-G (Active) ${ }^{9}$ with wire loop deposits, hyaline thrombi, diffuse endocapillary proliferation, and extravasated red cells suggestive for thrombotic microangiopathy. Activity levels for ADAMTS-13 (A Disintegrin And Metalloproteinase with a Thrombospondin Type 1 Motif, Member 13), however, were normal at $99 \%$ (normal $\geq 67 \%$ ). Admission urine spot Protein:Creatinine ratio was 3.52 - consistent with nephrotic range proteinuria.

Complete blood count showed white blood cell count (WBC) of $4.1 \mathrm{~K} / \mathrm{mcL}(4.0-10.5)$, hemoglobin $(\mathrm{Hb})$ of $8.7 \mathrm{~g} / \mathrm{dL}(11.5-15.0)$, hematocrit (Hct) of $25.2 \%(34.0-44.0)$, and platelet count (Plt) of $50 \mathrm{~K} / \mathrm{mcL}$ (150-400). Lactate dehydrogenase was elevated at $341 \mathrm{U} / \mathrm{L}$ (140-271), haptoglobin suppressed at $<30$ $\mathrm{mg} / \mathrm{dL}$ (44-215), and Total bilirubin was normal at $0.5 \mathrm{mg} / \mathrm{dL}(0.3-1.0)$. Direct Antiglobulin Test (DAT) was weakly positive using both IgG and C3d specific antihuman globulin (AHG); the eluate was negative. Schistocytes at approximately 2-3 per high power field were noted on the peripheral smear. Coagulation tests showed normal results for Prothrombin Time (PT), International Normalized Ratio (INR), Partial Thromboplastin Time (PTT), and Fibrinogen (Fib). D-Dimer was non-elevated. Studies for lupus anticoagulant, anticardiolipin IgG and IgM, Beta 2 Glycoprotein 1 IgG and IgM were negative.

Given her history of cough, dyspnea, and abdominal discomfort with tenderness to palpation, computed tomography scanning of the chest, abdomen, and pelvis was obtained. Trace pericardial and bilateral pleural effusions were noted. Prominent axillary and mediastinal lymph nodes were felt to be hyperplastic in nature and consistent with active SLE. Minimal interstitial pulmonary edema was noted as well as subtle, peripherally distributed ground glass micronodules suggestive for an infectious process, prompting empiric antibiotic therapy. A ventilation perfusion scan was low-probability for pulmonary embolus. Echocardiogram was unremarkable with normal systolic function and no significant valvular vegetations.

There was no improvement during initial treatment with empiric antibiotics, high-dose corticosteroids, and mycophenolic acid with progression of renal failure to a peak $\mathrm{Cr}$ of 2.5 $\mathrm{mg} / \mathrm{dL}$ by day 16 and proteinuria (by spot urine Pr:Cr ratio) to a peak of $11.55 \mathrm{mg} / \mathrm{mg}$ by day 19 (Figure 1). In addition, a significant progression in her microangiopathic hemolytic picture occurred, with development of red cell transfusion dependence and hemolysis to a nadir hemoglobin of $6.4 \mathrm{~g} / \mathrm{dL}$ by hospital day 10 and nadir platelet count of $21 \mathrm{~K} / \mathrm{mcL}$ by day 13 . This was accompanied by a rise in the $\mathrm{LDH}$ to peak of $1689 \mathrm{U} / \mathrm{L}$ on day 12 . Concern existed for intercurrent mycophenolic acid-mediated cytopenias, so this drug was stopped. To address the lupus nephritis, cyclophosphamide was administered on Day 14.

Plasma-based therapy was initially withheld given absence of microthrombi on preliminary (but not final; Figure 2) renal biopsy report. This was felt to be justified with return of ADAMTS-13 activity level at 99\%. However, after further multi-disciplinary discussion, it was decided that a therapeutic trial of plasma exchange would proceed following first dose of rituximab (with appropriate window to prevent premature apheresis removal of rituximab, which was added as a salvage agent for lupus nephritis) ${ }^{10}$ with plan to convert to eculizumab if unresponsive. An atypical HUS panel, involving sequencing and analysis of the exonic regions of 12 genes: $C F H, M C P$ (CD46), CFI, C3, CFB, CFHR1, CFHR3, CFHR4, CFHR5, thrombomodulin, plasminogen, DGKE - was negative for disease associated mutations.

Hemolysis and red cell transfusion requirement continued in the face of plasma exchange therapy, but platelet counts were responsive. Creatinine declined during plasma exchange but it was unclear what proportion of this decline represented a therapeutic response and what proportion represented a plasmapheresis removal effect. Rituximab dose 2 (day 30) and cyclophosphamide dose 2 (day 34) were applied with planned pauses in the apheresis regimen to maximize immunosuppressive agent dwell time. These pauses were associated with immediate declines in the platelet count. Additionally, proteinuria did not appreciably respond to the plasma exchange course with a spot urine Pr:Cr ratio of $10.84 \mathrm{mg} / \mathrm{mg}$ on hospital day 50 , following 18 rounds of plasma exchange.

Plasma exchange was also declared ineffective at controlling the microangiopathic picture. Following meningo- and pneumo-coccal vaccination and institution of antibiotic prophylaxis against meningococcal infection, Eculizumab was initiated on day 49. Eculizumab induced immediate reduction in hemolytic pace allowing spontaneous recovery of hemoglobin levels. Platelet counts, which had been rising in response to plasma exchange, demonstrated a pronounced increment after eculizumab administration followed by progressive recovery thereafter. She was discharged following the second eculizumab dose on day 56 .

Haptoglobin, which had been absolutely suppressed throughout her hospitalization, again became detectable following 5 weekly doses (900 mg IV 4 then $1200 \mathrm{mg}$ IV week 5) by day 83 signaling complete resolution of the hemolytic process. In addition, significant
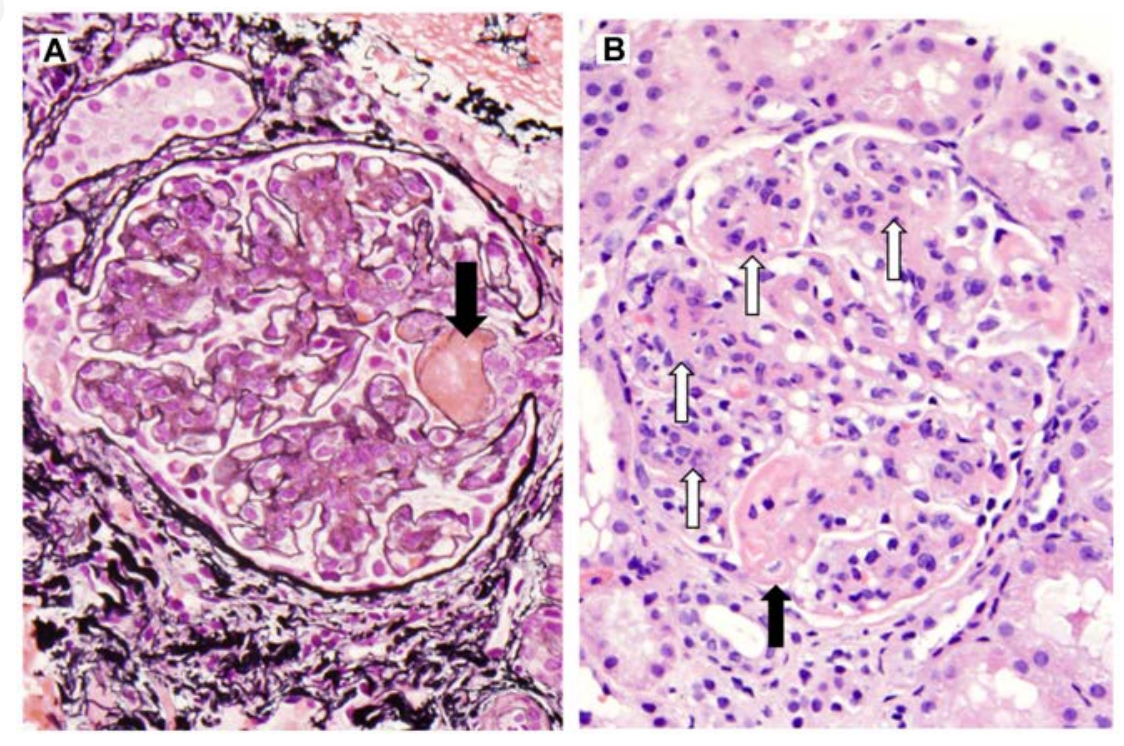

Figure 1. Renal biopsy images demonstrating the presence of Class IV G (active) lupus nephritis. A) Jones Silver stain demonstrating fibrin thrombus (arrow) within a hilar arteriole; B) Hematoxylin \& Eosin stain demonstrating diffuse endocapillary hypercellularity (white arrows) and wire loops (black arrow). 
renal recovery occurred during Eculizumab therapy with improvement in BUN, $\mathrm{Cr}$ and urine $\operatorname{Pr}: C r$ to $25 \mathrm{mg} / \mathrm{dL}, 1.1 \mathrm{mg} / \mathrm{dL}$, and 1.64 $\mathrm{g} /$ day also by day 83 . Her proteinuria continued to improve with spot urine Pr:Cr ratio of 0.65 $\mathrm{mg} / \mathrm{mg}$ by day 160 . At last follow up, day 226 eculizumab maintenance had continued to repress hemolytic activity with $\mathrm{Hb} 11.0 \mathrm{~g} / \mathrm{dL}$, platelet count $306 \mathrm{~K} / \mathrm{mcL}$, Cr $0.9 \mathrm{mg} / \mathrm{dL}$, and spot urine Pr:Cr 0.46 mg/mg.

\section{Discussion}

We describe a case of complement mediated TMA with lupus nephritis as the apparent driver in a patient with negative aHUS gene panel and partial (platelets only) response to therapeutic plasma exchange. Important aspects of this case include the continued hemolysis and transfusion requirement in the face of daily plasma exchange as well as the requirement for at least 5 weekly doses of eculizumab prior to complete cessation of hemolysis.

Our patient demonstrated a significant degree of hemolytic involvement with resolution of haptoglobinemia only after multiple (in our case 5) doses of eculizumab. Coppo et al., ${ }^{11}$ reiterate this experience in their case report of a child with diffuse proliferative lupus nephritis also associated with significant proteinuria (with spot urine Pr:Cr as high as $10 \mathrm{mg} / \mathrm{mg}$ ) and atypical HUS whose haptoglobinemia did not improve until after 3 doses of eculizumab. As with our case, theirs demonstrated exquisite platelet responsiveness to eculizumab, a negative aHUS genetic screening panel, and significant improvement in renal function and proteinuria.

We noted a selective response to plasma exchange in our patient - platelet counts, and possibly creatinine improved, but hemolysis was unresponsive. A very similar patient, also with diffuse proliferative lupus nephritis, Class IV $\mathrm{G}$ with nephrotic-range proteinuria (4 $\mathrm{g} /$ day) and detectable ADAMTS-13 (33\%) activity did respond to treatment with therapeutic plasma exchange. ${ }^{12}$ Interestingly, response did not appear to accompany the initial $5 / 12$ plasma exchange procedures in which albumin was used as the primary exchange media but did appear during subsequent procedures during which plasma was used as the exchange media. A genetic aHUS panel or testing for anti FH antibodies was not performed. Speculatively, an anti-FH antibody was proposed.

Atypical HUS due to Factor $\mathrm{H}$ antibodies constitutes the only Category I (i.e., first-line therapy) indication for plasma exchange in atypical HUS. ${ }^{13}$ The 2013 American Society for Apheresis evidence-based guidelines classify aHUS $^{13}$ due to complement gene mutations as a Category II indication (i.e., second-line therapy) and aHUS due to MCP mutations as a Category IV indication (i.e., not indicated). One wonders why such a dramatic response in Samson et al occurred only after conversion to plasma as the replacement media. Rather than a possible Factor $\mathrm{H}$ antibody, it stands to reason that the patient instead bore a dysfunctional complement regulatory protein that was replaced by addition of donor plasma to the treatment regimen.

El-Husseini et al. ${ }^{14}$ report a similar patient with steroid-refractory lupus nephritis and hypocomplementemia in whom microangiopathic hemolytic anemia developed. ADAMTS13 was detectable and a limited aHUS panel was negative. Hemolysis continued despite treatment with 8 rounds of therapeutic plasma exchange but both renal and hematologic responses were noted following initiation of eculizumab.

Interestingly, eculizumab may also bear value in the setting of recalcitrant, non-TMA lupus nephritis. Pickering and colleagues report a patient in whom Class IV-G (A/C) lupus nephritis involving hypocomplementemia and nephrotic range proteinuria refractory to multiple rounds of cyclophosphamide, rituximab, mycophenolate mofetil, and tacrolimus responded to eculizumab ther- apy. ${ }^{15}$ Notably, renal function and proteinuria responded dramatically following initiation of eculizumab therapy (4 weekly doses of 1200 $\mathrm{mg}$ followed by two $1200 \mathrm{mg}$ doses every 2 weeks).

The connection between complement activation and thrombotic processes involved during microangiopathy has recently been explored. Binding of $\mathrm{C} 3 \mathrm{a}$ and $\mathrm{C} 5 \mathrm{a}$ to their respective receptors leads to activation of endothelial membranes and enhanced expression of adhesion molecules and secretion of Von Willebrand Factor and P-selectin with subsequent platelet binding and activation. Concomitantly, downregulation of surface thrombomodulin expression further enhances the prothrombotic phenotype by impairing the Protein C pathway. Receptors for C3a and C5a also appear on platelets leading to platelet activation upon complement binding. . $^{16,17}$

An aHUS panel, performed in our patient by an outside laboratory specializing in aHUS diagnostics, was negative. The presence of either a known-but-untested or an as-yet undescribed genetic complement abnormality remains possible. In two large series of patients with both familial and sporadic aHUS, the absence of detectable genetic abnormalities was reported in $\sim 25 \%$ to $48 \%$ of individuals. ${ }^{5,6}$

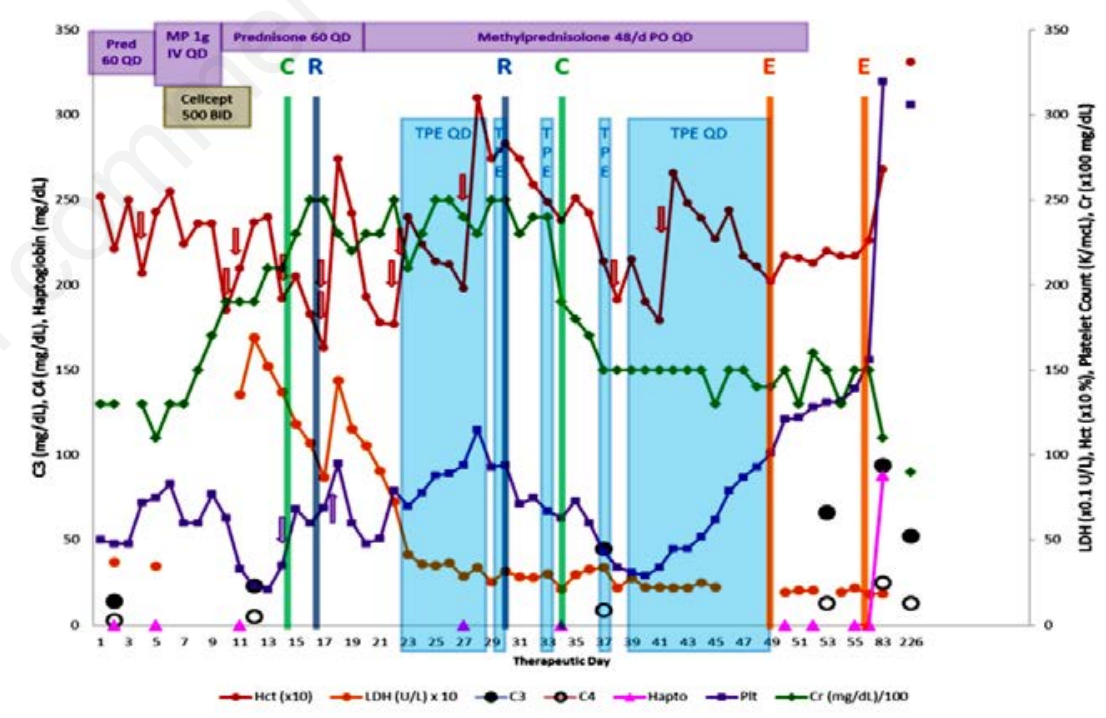

Figure 2. Therapeutic course. Horizontal lavender boxes depict steroids [and hydroxychloroquine $(H Q)$ course; $P O=$ Prednisone dose; $M P=$ Methylprednisolone, 1 gram]. $M$ = Mycophenolate Mofetil; $R$ = Rituximab (1000 mg IV); C = Cyclophosphamide (C1 500 mg IV; C2 800 mg IV); E = Eculizumab; PE = Therapeutic Plasma Exchange; QD = daily; IV = Intravenous. Note that platelet counts were responsive to TPE but hemolysis-engendering-transfusion (downward pointing arrows, each one representing a unit of RBCs) continued in the face of continued plasma exchange. Eculizumab was dosed on days 49 (900 mg IV shown), 56 (900 mg IV shown), and 63 (900 mg IV, not shown), 70 (900 mg IV, not shown), and 77 (1200 mg IV, not shown) leading up to day 83 repeat laboratory testing. Eculizumab (1200 mg IV every 14 days) has been continued thereafter. Detectability of haptoglobin (and hence, cessation of hemolysis) was not established until after 5 doses of eculizumab, at which time the creatinine had finally normalized. 
The tendency toward unchecked complement activation following sometimes innocuous triggers, such as upper respiratory tract infection, gastroenteritis, or pregnancy, ${ }^{5,6}$ may initiate a potentially fatal microangiopathic process that ultimately leads to end stage renal disease in over $50 \%$ and death in over $30 \%$ of affected individuals. ${ }^{5}$ While an alternative driver - such as infection - was certainly possible in our patient, there was not an appreciable response with empiric antibiotics and the overall clinical presentation appeared most consistent with systemic lupus erythematosus disease activity.

We therefore conclude that the primary driver for complement activation - as evidenced by hypocomplementemia persisting even in the face of daily receipt of donor plasma (a source of donor complement) during therapeutic apheresis - was a renally focused (lupus nephritis) nidus of intense immunologic activity. Unfortunately, the immunofluorescence samples in our case were corrupted thus no immunofixation results exist. Initiation of eculizumab to halt what was a secondary consequence of primary lupus nephritis proved effective.

Once on eculizumab, it is difficult to know when the medication can be discontinued. In many instances, maintenance treatment is continued indefinitely; an important factor given estimated annual drug costs of $\$ 350,000$ to $\$ 645,000 .{ }^{17}$ Early reports suggest that maintenance dosing may be modified, and in some cases even suspended, provided complement activity remains suppressed and patients are carefully surveilled (using home urine dipstick for hemoglobinuria and periodic laboratory testing for markers of hemolysis and schistocytosis) to detect relapse, which would require immediate reinstitution of eculizumab. ${ }^{17,18}$ Gatault et al., studied the use of eculizumab trough levels finding that elimination half-life varied significantly based upon weight with an increase from 7.8 days in $100 \mathrm{~kg}$ patient to 19.5 days in a $40 \mathrm{~kg}$ patient. ${ }^{19,20}$ Preliminary data, therefore, suggested that $1200 \mathrm{mg}$ maintenance doses could be spaced to every 4 weeks in patients $<90 \mathrm{~kg}$ and every 6 weeks in patients $<70 \mathrm{~kg}$ but that additional studies involving individualized dosing were necessary.

In conclusion, our case emphasizes the need for a high degree of clinical awareness surrounding complement-mediated TMA, as well as lupus nephritis as a primary driver.
Results for ADAMTS-13 activity and aHUS gene panels may be non-diagnostic, but hypocomplementemia is an important clue. Renal outcomes in the setting of complementmediated TMA, even in the setting of intercurrent hematologic responses, are typically poor. Eculizumab, although expensive, is highly effective at inducing both hematologic and renal responses. Although plasma-based therapeutics can be initiated, clinicians should have a low threshold to move on to late-complement inhibition in the face of non-response - particularly if the ADAMTS-13 activity level is detectable. Although experience is growing in terms of recognition of complement-mediated TMA, additional studies are required to more clearly identify optimal eculizumab dosing, treatment schedules, monitoring, and endpoints for therapy.

\section{References}

1. Constantinescu AR, Bitzan M, Weiss LS, et al. Non-enteropathic hemolytic uremic syndrome: causes and short-term course. Am J Kidney Dis 2004;43:976-82.

2. Loirat C, Fremeaux-Bacchi V. Atypical hemolytic uremic syndrome. Orphanet J Rare Dis 2011;6:60.

3. George JN, Nester CM. Syndromes of thrombotic microangiopathy. New Engl J Med 2014;371:654-66.

4. Davin, JC, van de Kar NCAJ. Advances and challenges in the management of complement-mediated thrombotic microangiopathies. Ther Adv Hematol 2015;6:17185.

5. Noris M, Caprioli J, Bresin E, et al. Relative role of genetic complement abnormalities in sporadic and familial aHUS and their impact on clinical phenotype. Clin J Am Soc Nephrol 2010;5:1844-59.

6. Sellier-Leclerc AL, Fremeaux-Bacchi V, Dragon-Durey MA, et al. Differential impact of complement mutations on clinical characteristics in atypical hemolytic uremic syndrome. J Am Soc Nephrol 2007;18:2392-400.

7. Nester CM, Barbour T, Rodriguez de Cordoba S, et al. Atypical HUS: state of the art. Mol Immunol 2015;67:31-42.

8. Legendre CM, Licht C, Muus $\mathrm{P}$, et al. Terminal complement inhibitor eculizumab in atypical hemolytic-uremic syndrome.
N Engl J Med 2013;368:2169-81.

9. Weening JJ, D'Agati VD, Schwartz MM, et al. The classification of glomerulonephritis in systemic lupus erythematosis revisited. J Am Soc Nephrol 2004;15:241-50.

10. Gunnarsson I, Jonsdottir T. Rituximab treatment in lupus nephritis - where do we stand? Lupus 2013;22:381-9.

11. Coppo R, Peruzzi L, Amore A, et al. Dramatic effects of eculizumab in a child with diffuse proliferative lupus nephritis resistant to conventional therapy. Pediatr Nephrol 2015;30:167-72.

12. Samson M, Audia S, Leguy V, et al. Haemolytic-uraemic syndrome during severe lupus nephritis: efficacy of plasma exchange. Int Med J 2012;42:95-8.

13. Schwartz J, Winters JL, Padmanabhan A, et al. Guidelines on the use of therapeutic apheresis in clinical practice. Evidencebased approach from the Writing Committee of the American Society for Apheresis: the sixth special issue. J Clin Apheresis 2013;28:145-284.

14. El-Husseini A, Hannan S, Awad A, et al. Thrombotic microangiopathy in systemic lupus erythematosus: efficacy of eculizumab. Am J Kidney Dis 2015;65:127-30.

15. Pickering MC, Ismajli M, Condon MB, et al. Eculizumab as rescue therapy in severe resistant lupus nephritis. Rheumatology (Oxford) 2015;54:2286-8.

16. Morigi M, Galbusera M, Gastoldi S, et al. Alternative pathway activation of complement by shiga toxin promotes exuberant c3a formation that triggers microvascular thrombosis. J Immunol 2011;187:172-80.

17. Conway EM. HUS and the case for complement. Blood 2015;126:2085-90.

18. Cugno M, Gualtierotti R, Possenti I, et al. Complement functional tests for monitoring eculizumab treatment in patients with atypical hemolytic uremic syndrome. J Thromb Haemost 2014;12:1440-8.

19. Ardissino G, Testa S, Possenti I, et al. Discontinuation of eculizumab maintenance treatment for atypical hemolytic uremic syndrome: a report of 10 cases. Am J Kidney Dis 2014;64:633-7.

20. Gatault P, Brachet G, Ternant D, et al. Therapeutic drug monitoring of eculizumab: rationale for an individualized dosing schedule. MAbs 2015;9:1205-11. 